\title{
Analysis of time-resolved argon line spectra from OMEGA direct-drive implosions
}

Cite as: Rev. Sci. Instrum. 79, 10 E310 (2008); https://doi.org/10.1063/1.2965779

Submitted: 20 May 2008. Accepted: 09 July 2008. Published Online: 31 October 2008

R. Florido, T. Nagayama, R. C. Mancini, R. Tommasini, J. A. Delettrez, S. P. Regan, V. A. Smalyuk, R. Rodríguez, and J. M. Gil

ARTICLES YOU MAY BE INTERESTED IN

Direct-drive inertial confinement fusion: A review

Physics of Plasmas 22, 110501 (2015); https://doi.org/10.1063/1.4934714

Characterization of direct-drive-implosion core conditions on OMEGA with time-resolved Ar K-shell spectroscopy

Physics of Plasmas 9, 1357 (2002); https://doi.org/10.1063/1.1456530

Time-resolved characterization and energy balance analysis of implosion core in shockignition experiments at OMEGA

Physics of Plasmas 21, 102709 (2014); https://doi.org/10.1063/1.4898329

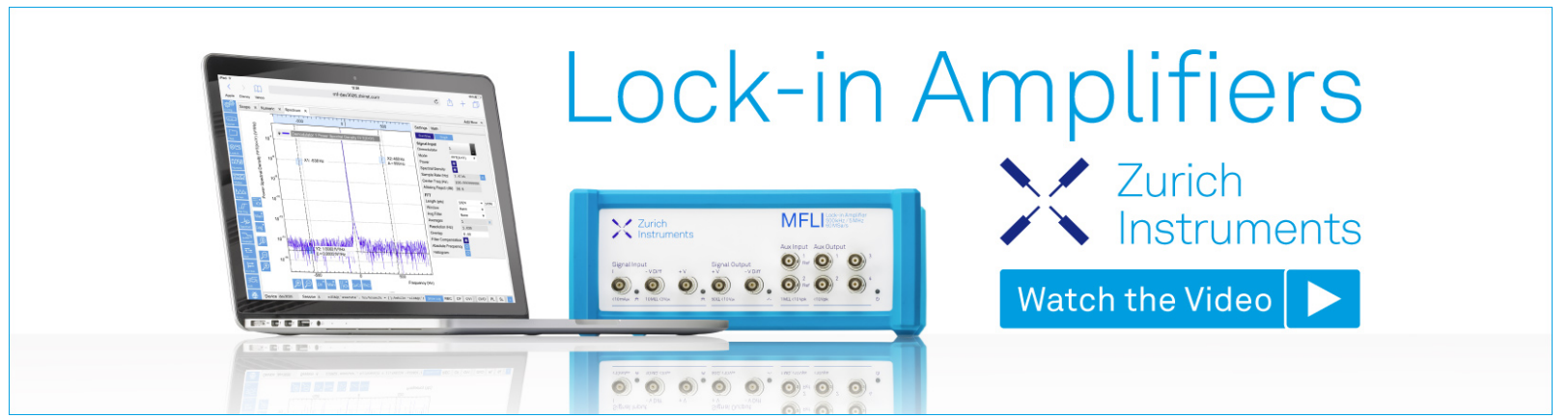

Rev. Sci. Instrum. 79, $10 E 310$ (2008); https://doi.org/10.1063/1.2965779 


\title{
Analysis of time-resolved argon line spectra from OMEGA direct-drive implosions ${ }^{\text {a) }}$
}

\author{
R. Florido, ${ }^{1, b)}$ T. Nagayama, ${ }^{1}$ R. C. Mancini, ${ }^{1}$ R. Tommasini, ${ }^{2}$ J. A. Delettrez, ${ }^{3}$ \\ S. P. Regan, ${ }^{3}$ V. A. Smalyuk, ${ }^{3}$ R. Rodríguez, ${ }^{4}$ and J. M. Gil ${ }^{4}$ \\ ${ }^{1}$ Department of Physics, University of Nevada, Reno, Nevada 89557, USA \\ ${ }^{2}$ Lawrence Livermore National Laboratory, Livermore, California 94550, USA \\ ${ }^{3}$ Laboratory for Laser Energetics, University of Rochester, New York 14623, USA \\ ${ }^{4}$ Departamento de Física, Universidad de Las Palmas de Gran Canaria, \\ 35017 Las Palmas de Gran Canaria, Spain
}

(Presented 12 May 2008; received 20 May 2008; accepted 9 July 2008; published online 31 October 2008)

We discuss the observation and data analysis of argon $K$-shell line spectra from argon-doped deuterium-filled OMEGA direct-drive implosion cores based on data recorded with two streaked crystal spectrometers. The targets were $870 \mu \mathrm{m}$ in diameter, $27 \mu \mathrm{m}$ wall thickness plastic shells filled with $20 \mathrm{~atm}$ of deuterium gas, and a tracer amount of argon for diagnostic purposes. The argon $K$-shell line spectrum is primarily emitted at the collapse of the implosion and its analysis provides a spectroscopic diagnostic of the core implosion conditions. The observed spectra includes the He $\alpha$, $\operatorname{Ly} \alpha, \operatorname{He} \beta, \operatorname{He} \gamma, \operatorname{Ly} \beta$, and $\operatorname{Ly} \gamma$ line emissions and their associated He- and Li-like satellites thus covering a broad photon energy range from 3100 to $4200 \mathrm{eV}$ with a spectral resolution power of approximately 500. The data analysis relies on detailed atomic and spectral models that take into account nonequilibrium collisional-radiative atomic kinetics, Stark-broadened line shapes, and radiation transport calculations. (C) 2008 American Institute of Physics. [DOI: 10.1063/1.2965779]

\section{INTRODUCTION}

Diagnosing the temperature and density plasma conditions of implosion cores is of current interest in inertial confinement fusion (ICF) experiments. ${ }^{1-4}$ We report here on the determination of time-resolved and spatially averaged plasma conditions from direct-drive experiments performed at the Laboratory for Laser Energetics' OMEGA laser facility. Electron temperature and density are determined spectroscopically from the analysis of $K$-shell argon x-ray line spectra recorded with streaked crystal spectrometers. The spectroscopic analysis relies on the electron temperature and density sensitivity of the argon line emission through the density and temperature dependence of the atomic level populations and the density sensitivity of the Stark broadening effect of the line shapes. A similar spectroscopic diagnostic has been applied to indirect-drive ICF experiments ${ }^{1,3}$ and direct-drive implosions that used thinner wall $(20 \mu \mathrm{m})$ plastic targets. ${ }^{2,4}$ Here, we apply it to thicker plastic shells that have a wall thickness of $27 \mu \mathrm{m}$ and a deuterium filling pressure of $20 \mathrm{~atm}$. In addition, the atomic and radiation physics model employed to analyzed the data includes Starkbroadened line shapes for satellite line transitions up to principal quantum number $n=4$, and the radiative recombination emission that results from the radiative recombination process from $\mathrm{H}$ - into He-like argon ground states.

\footnotetext{
${ }^{\text {a) }}$ Contributed paper, published as part of the Proceedings of the 17th Topical Conference on High-Temperature Plasma Diagnostics, Albuquerque, New Mexico, May 2008.

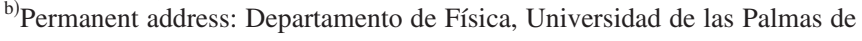
Gran Canaria, 35017 Las Palmas de Gran Canaria, Spain.
}

\section{EXPERIMENTAL DETAILS AND DATA}

The direct-drive experiments discussed here consisted of imploding spherical plastic microballoon targets driven with the OMEGA laser system. The microballoons had an initial diameter of $435 \mu \mathrm{m}$, a plastic wall thickness of $27 \mu \mathrm{m}$, and an outer Al layer of $0.1 \mu \mathrm{m}$. They were filled with $20 \mathrm{~atm}$ of $\mathrm{D}_{2}$ and $0.072 \mathrm{~atm}$ of Ar, which was used as the spectroscopic diagnostic, and they were driven with $1 \mathrm{~ns}$ squared OMEGA laser pulses. The UV energy on target was $23 \mathrm{~kJ}$ and laser beam smoothing included distributed phase plates, smoothing by spectral dispersion and distributed polarization rotators.

Radiation emission from the argon tracer becomes bright at the collapse of the implosion when the deuterium gas reaches keV-range electron temperatures and the electron density order of magnitude is $10^{24} \mathrm{~cm}^{-3}$. The argon radiation emission includes intense $K$-shell x-ray line transitions that span the photon energy range from 3000 to $4200 \mathrm{eV}$. The $\mathrm{x}$-ray spectrum was recorded with two streaked crystal spectrometers: SSC1 and SSCA. SSC1 was used in a low-speed mode (sweep speed $150 \mathrm{ps} / \mathrm{mm}$ ) to survey the X-ray emission over a broad time interval while SSCA was used in a high-speed mode (sweep speed $50 \mathrm{ps} / \mathrm{mm}$ ) to unfold the details of the time history of argon line emission from the core through the collapse of the implosion. The raw data were recorded on film and processed with an IDL code to account for film density to intensity conversion, Be filter transmission, and crystal reflectivity correction. Figure 1 displays examples of the data recorded for OMEGA shot 47477. The increased sweep speed of SSCA relative to that of SSC1 is 


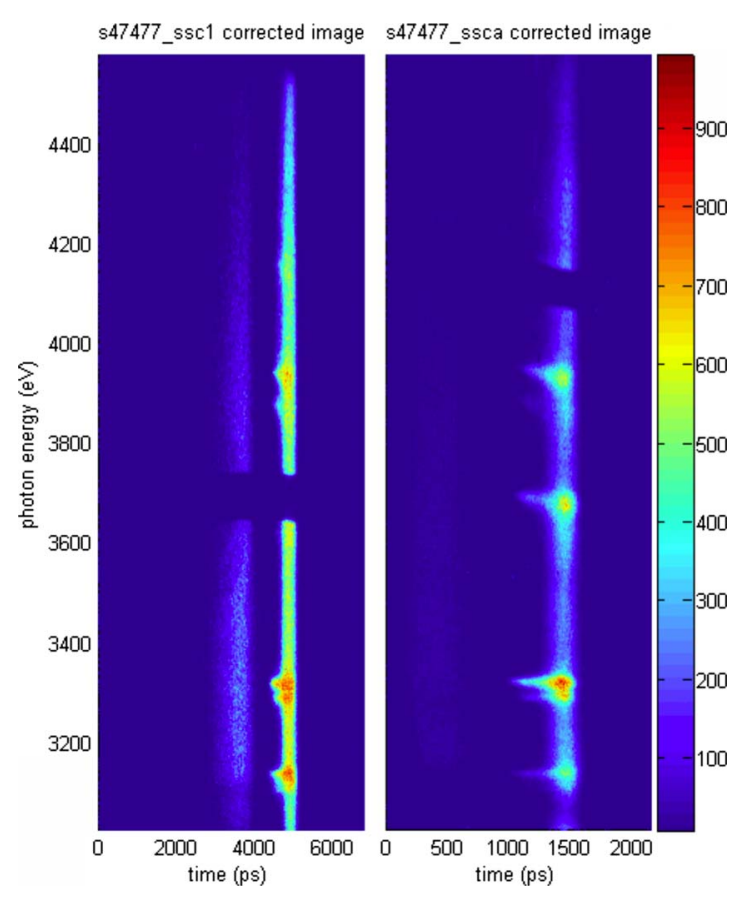

FIG. 1. (Color online) Image data recorded with streaked crystal spectrometers SSC1 and SSCA in OMEGA shot 47477.

clearly observed in the image data. We note that the larger time window afforded by the low-speed mode of SSC1 shows continuum radiation emission from plastic plasma blow-off early in time and characteristic argon line emission late in time (i.e., at the collapse of the implosion). On the other hand, the larger time resolution of SSCA data shows the gradual increment of line broadening effect as the core goes through stagnation. With reference to the image data in Fig. 1, horizontal lineouts represent time histories of radiation emitted within a given photon energy range while vertical lineouts represent time-resolved spectra. An example of a time-resolved spectrum extracted from the image data of SSCA is displayed is Fig. 2. The spectral resolution power $(\lambda / \Delta \lambda)$ is approximately 500 . Several line transitions of Heand $\mathrm{H}$-like Ar ions have been identified and labeled in the spectrum, namely $\operatorname{He} \alpha 1 s^{2}-1 s 2 p, \operatorname{Ly} \alpha 1 s-2 p, \operatorname{He} \beta 1 s^{2}-1 s 3 p$, $\operatorname{He} \gamma 1 s^{2}-1 s 4 p$, and Ly $\beta 1 s-3 p$. Weaker, and sometimes

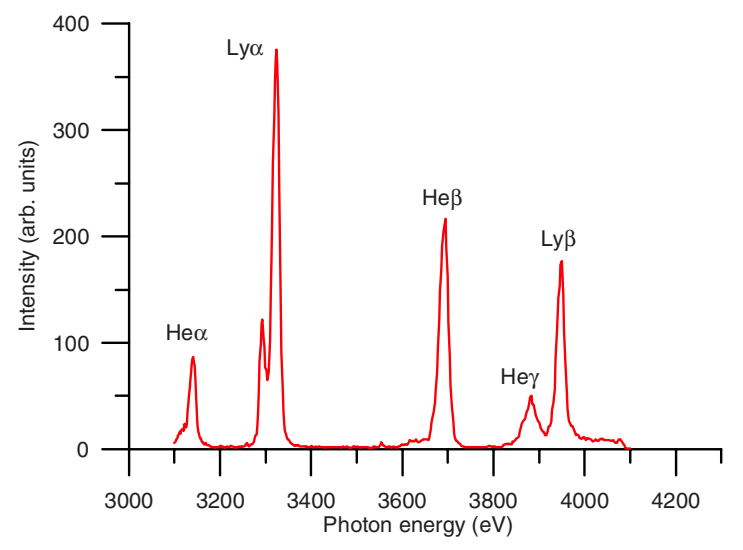

FIG. 2. (Color online) Time-resolved argon $K$-shell line spectrum integrated over a subinterval of 50 ps recorded with SSCA in OMEGA shot 47477. Several line emissions in $\mathrm{He}$ - and $\mathrm{H}-$ like $\mathrm{Ar}$ ions are labeled in the plot. heavily blended with parent lines, satellite line transitions arising from autoionizing states in $\mathrm{He}-$ and Li-like Ar ions are also present in the data.

\section{SPECTROSCOPIC DETERMINATION OF TEMPERATURE AND DENSITY TIME HISTORIES}

A set of time-resolved (contiguous) spectra was extracted from the SSCA image data covering the broad time interval from early to late times. Each spectra lineout was integrated over a time subinterval of 50 ps. This subinterval represents a compromise between good signal-to-noise ratio in the spectrum and a subinterval of integration small enough to afford good time resolution to describe the time history of electron temperature and density through the collapse (stagnation) of the implosion. The analysis of the spectrum uses the detailed collisional-radiative atomic kinetics model and code ABAKO. ${ }^{5}$ For this application, an ABAKO model was constructed including all ionization stages from $\mathrm{C}$-like $\mathrm{Ar}$ through the fully stripped ion including all nonautoionizing and autoionizing states consistent with continuum lowering. ${ }^{6}$ For the temperature and density conditions studied here this means that states with principal quantum number $n$ up to 4 or 5 are typically included in the model. This permits accounting for the effects of high order satellites that overlap heavily with the parent line transition thus affecting both the emissivity and opacity of composite spectral features. ${ }^{7}$ The populations in ABAKO are computed as a result of the population and depopulation of atomic levels driven by several atomic processes including electron collisional excitation and deexcitation, electron collisional ionization and recombination, autoionization and resonant electron capture, spontaneous radiative decay, and radiative recombination. Radiation transport effects due to line trapping in the plasma source are taken into account via escape factors ${ }^{8}$ and the emergent line intensity distribution is computed using an analytical integration of the radiation transport equation for the case of a uniform spherical plasma. ${ }^{9}$ A plasma model of uniform distribution of temperature and density is employed in the analysis since the $\mathrm{x}$-ray spectrometers used in the experiments recorded time resolved but spatially integrated line spectra and thus there is not enough information in the data to uniquely unfold the spatial structure. Detailed Stark-broadened line shapes play an important role in the analysis of the implosion core spectra. A data base of line profiles was computed for this application including the broadening effects due to plasma electron and ion microfields, as well as natural and Doppler broadening. Furthermore, since deuterium ions are the dominant type of perturbing ion in the plasma core, ion dynamics effects were also considered in the Stark line broadening calculation. ${ }^{10}$ Thus, detailed Stark-broadened line profiles have been included for line transitions arising from excited states as well as satellite line transitions originating from autoionizing states. As an illustration of the data analysis, Fig. 3 shows a comparison between experimental and theoretical spectra based on a weighted least-squares minimization. A broad data base of electron temperature and density cases was considered for this procedure that yields a single, absolute minimum from which the conditions for the best comparison are extracted. The goodness of the fit is 


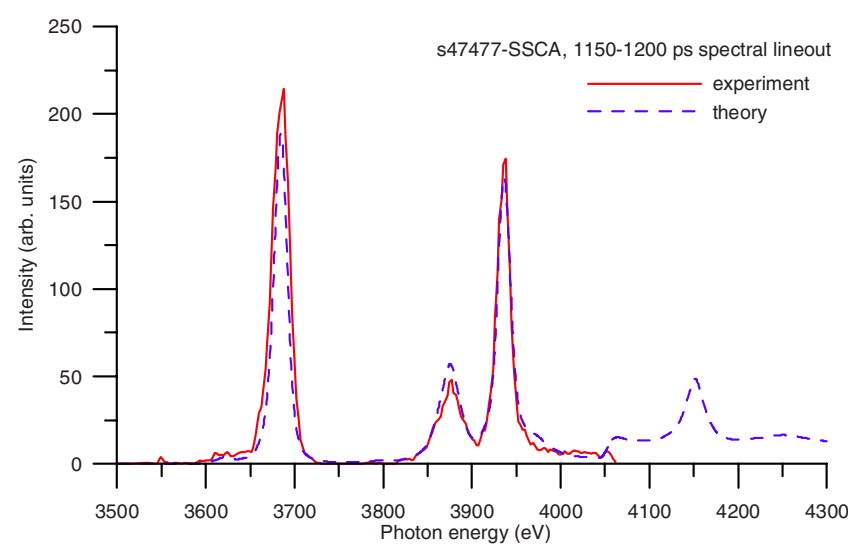

FIG. 3. (Color online) Comparison between theory (dashed curve) and data (solid curve) of argon $K$-shell line spectrum over the spectral region of $\mathrm{He} \beta$, $\mathrm{He} \gamma$, and Ly $\beta$ line transitions. The comparison is based on a weighted least-squares-minimization procedure; best fit parameters are $T_{e}=1405 \mathrm{eV}$ and $N_{e}=5.2 \times 10^{23} \mathrm{~cm}^{-3}$.

assessed by the value of the weighted $Q^{2}$ divided by the number of degrees of freedom which is of order 1. A systematic application of this analysis to each of the lineouts in the series results in a time history of electron temperature $T_{e}$ and density $N_{e}$ through the collapse of the implosion. Figure 4 displays the temperature and density time histories obtained for OMEGA shot 47477. Early in the implosion core col-

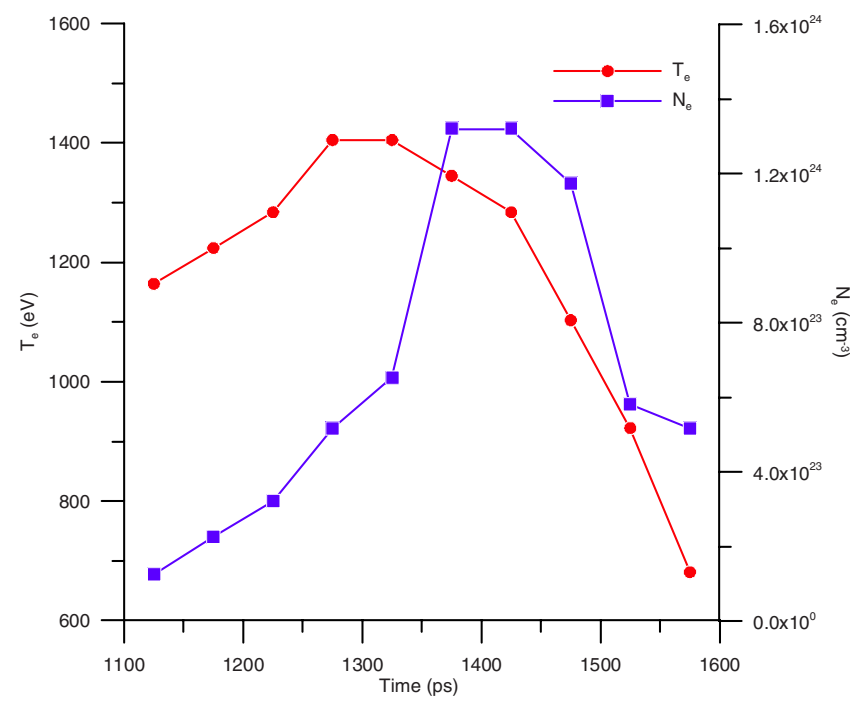

FIG. 4. (Color online) Time history of electron temperature $T_{e}$ (circles) and density $N_{e}$ (squares) extracted from the analysis of SSCA data from OMEGA shot 47477 through the collapse of the implosion. lapse both temperature and density are increasing. The temperature reaches a maximum of approximately $1400 \mathrm{eV}$ and then starts to drop. The density continues to increase after the temperature reaches its maximum, eventually peaking at $1.3 \times 10^{24} \mathrm{~cm}^{-3}$. We note that since the fraction of argon ions in the core is very small the electron number density of electrons is approximately equal to the number density of deuterium ions, i.e., to within $4 \%$, and thus can also be used to extract the mass density.

\section{CONCLUSIONS}

We have applied a detailed atomic kinetics, line shape and radiation physics model to the analysis of time-resolved $\mathrm{x}$-ray line spectra emitted by an argon spectroscopic tracer added to a deuterium-filled implosion core. Based on the electron temperature and density sensitivity of the line intensity distribution and the line shape broadening we have extracted the time history of temperature and density through the collapse of the implosion. Beyond this application case to thick-wall $(27 \mu \mathrm{m})$ plastic shell targets, work is in progress to apply this spectroscopic analysis to the spectra recorded in low-adiabat implosion cores which have been designed to optimize implosion core compression.

\section{ACKNOWLEDGMENTS}

This work was supported by DOE/NLUF Grant No. DE-FG52-07NA28062 and LLNL.

${ }^{1}$ H. Nishimura, T. Kiso, H. Shiraga, T. Endo, K. Fujita, A. Sunahara, H. Takabe, Y. Kato, and S. Nakai, Phys. Plasmas 2, 2063 (1995).

${ }^{2}$ D. Haynes, Jr., D. T. Garber, C. F. Hooper, Jr., R. C. Mancini, Y. T. Lee, D. K. Bradley, J. Delettrez, R. Epstein, and P. A. Jaanimagi, Phys. Rev. E 53, 1042 (1996).

${ }^{3}$ N. Woolsey, B. A. Hammel, C. J. Keane, C. A. Back, J. C. Moreno, J. K. Nash, A. Calisti, C. Mosse, R. Stamm, B. Talin, A. Asfaw, L. S. Klein, and R. W. Lee, Phys. Rev. E 57, 4650 (1998).

${ }^{4}$ S. P. Regan, J. A. Delettrez, R. Epstein, P. A. Jaanimagi, B. Yaakobi, V. A. Smalyuk, F. J. Marshall, D. D. Meyerhofer, W. Seka, D. A. Haynes, I. E. Golovkin, and C. F. Hooper, Jr., Phys. Plasmas 9, 1357 (2002).

${ }^{5}$ R. Florido, Ph.D. thesis, Universidad de Las Palmas de Gran Canaria, 2007.

${ }^{6}$ J. C. Stewart and K. D. Pyatt, Astrophys. J. 144, 1203 (1966).

${ }^{7}$ I. E. Golovkin and R. C. Mancini, J. Quant. Spectrosc. Radiat. Transf. 65, 273 (2000).

${ }^{8}$ R. C. Mancini, R. F. Joyce, and C. F. Hooper, J. Phys. B 20, 2975 (1987).

${ }^{9}$ T. Burris-Mog, R. C. Mancini, J. E. Bailey, G. A. Chandler, G. Rochau, G. Dunham, P. W. Lake, K. Peterson, S. A. Slutz, T. A. Mehlhorn, I. E. Golovkin, and J. J. MacFarlane, J. Quant. Spectrosc. Radiat. Transf. 99, 120 (2006).

${ }^{10}$ R. C. Mancini, D. P. Kilcrease, L. A. Woltz, and C. F. Hooper, Jr., Comput. Phys. Commun. 63, 314 (1991). 